\title{
Dynamic Scheduling for Scalable Media Transmission over cdma2000 1xEV-DO Broadcast and Multicast Networks
}

\author{
Kyungtae $\mathrm{Kang}^{1}$, Jinsung $\mathrm{Cho}^{2, \star}$, Yongwoo $\mathrm{Cho}^{1}$, and Heonshik Shin ${ }^{1}$ \\ 1 School of Computer Science and Engineering, \\ Seoul National University, Seoul, 151-744, Korea \\ \{ktkang, xtg05, shinhs\}@cslab.snu.ac.kr \\ 2 Dept. of Computer Engineering, \\ Kyung Hee University, Youngin, 449-701, Korea \\ chojs@khu.ac.kr
}

\begin{abstract}
The cdma2000 1xEV-DO mobile communication system provides broadcast and multicast services (BCMCS) to meet an increasing demand for multimedia data services. Currently, broadcast and multicast streams are scheduled using a slot-based static algorithm, which fails to support dynamic environments where broadcast contents are added or removed on-line. We propose a dynamic packet-scheduling algorithm that works with a retransmission scheme to enable scalable and adaptive service across cdma2000 1xEV-DO BCMCS environments. Integrated with earliest deadline first (EDF) real-time scheduling, the proposed algorithm not only adapts efficiently to dynamic contexts but also satisfies the realtime requirements of broadcast streams. Furthermore, by exploiting the fine granular scalable (FGS) characteristics of the MPEG-4 Part 2 standard, our scheme can adapt to a changing resource environment more flexibly. Simulation results show a significant improvement in average playback quality.
\end{abstract}

Keywords: cdma2000 1xEV-DO BCMCS, earliest deadline first (EDF), resource allocation, fine granular scalable (FGS).

\section{Introduction}

Recently, work has begun, in both the Third Generation Partnership Project (3GPP) and in the 3GPP2 group, on enhancing 3G networks to support multimedia broadcast and multicast services. The 3GPP2 group recently baselined the specification for a cdma2000 high-rate broadcast packet-data air interface (BCMCS1] 1 2]. Their goal is to design a system that can deliver multimedia broadcast and multicast traffic while minimizing resource usage by both

\footnotetext{
* Corresponding author.

${ }^{1}$ In this paper, the term 'BCMCS' stands for cdma2000 1xEV-DO BCMCS.
} 
the radio access and core networks. In addition, users expect minimum latency when joining or leaving the network, and multimedia streams must be delivered continuously as mobile users move around. A hierarchical design with localized multicasting and local servers is necessary to provide a scalable system.

In this paper, we propose a dynamic scheduling algorithm combined with a retransmission scheme for the cdma2000 1xEV-DO BCMCS scheduler. The current static scheduling algorithm which is part of the BCMCS specification cannot adapt to an environment where content streams change dynamically. When a video stream is newly registered by mobile nodes, empty slots must be found to service this changed context. As the transmission rates of video streams differ from each other, the slot periods of these streams will also differ, which causes the serving slots to overlap. Also, if there are no slots left, the existing static scheduler is unable to service the new stream because it is impossible to adjust the bit-rate in a static scheme. Our proposed scheduling algorithm deals with such dynamic situations by exploiting the inherent attributes of the earliest deadline first (EDF) 3] algorithm. Our algorithm also works on the assumption that the video streams are fine granular scalable (FGS). By dynamically adjusting the scalability of the video streams that are already being serviced, new video streams can be admitted, although the playback quality is somewhat degraded. However the average playback quality degradation of all mobile nodes can be reduced compared to the current static scheme. Three policies to adjust the quality of each video stream are proposed, and their performance is compared experimentally. We also show how to control the admission of packets with a utilization bound test, using the EDF scheduling algorithm.

BCMCS currently employ Reed-Solomon coding to correct errors at the cost of air resources. If the channel condition is good, many slots will be overbooked due to the space taken by parity information, which reduces the number of empty slots available for a newly registered video stream. By adding a retransmission scheme to the EDF scheduling algorithm, we can save many slots when the condition of a channel is good, and guarantee the minimum playback quality by protecting the base-layer packets in FGS coding.

The remainder of this paper is organized as follows: in Section 2, we introduce our research background, and Section 3 gives the motivation for our proposed scheduling algorithm followed by a description. Simulation results are discussed in Section 4 and we conclude in Section 5.

\section{$2 \quad$ Background}

\subsection{Packet Scheduling in BCMCS}

In a unicast environment, 1xEV-DO employs a time-shared forward link that serves users sequentially in a time-multiplexed manner. The fundamental timing unit for forward link transmissions is a $1.67 \mathrm{~ms}$ slot that contains the pilot and MAC channels, and a data portion that may contain traffic or a control channel. The $1 \mathrm{xEV}$-DO system adopts a dynamic data rate transmission scheme which 
allows efficient data transmission with a data rate that varies according to the channel condition of each mobile node [4].

In a broadcast or multicast environment, however, a fixed transmission rate must be used, because it is impossible to reflect the link state of all mobile nodes. BCMCS video streams are delivered to one or more nodes in $1.67 \mathrm{~ms}$ time-slots that statically in advance the current static scheduler allocates. In receiving a cdma2000 1xEV-DO broadcast or multicast video stream, a mobile node obtains the content from the video server in pre-determined slots, but the service parameters are transmitted as an overhead message. When a mobile node requests a BCMCS video stream, packets are transmitted from the Packet Data Serving Node (PDSN) to the Packet Control Function (PCF), which uses a timestamp to make sure that the packet is received simultaneously at all base stations. The mobile node maintains its soft state by registering periodically. If no mobile node is left registered to it, the video stream is eventually terminated [5].

For error recovery, the broadcast MAC protocol does not use an ARQ-based error control scheme, because the same content will be delivered to many users simultaneously. Instead of handling retransmission requests individually, BCMCS use a Reed-Solomon forward error-correcting code. A Reed-Solomon encoder takes a block of digital data and adds extra 'check' bits. The encoded data is larger and therefore uses more physical slots, which means using more air resource. As a result, fewer video streams can be serviced than would be the case without Reed-Solomon coding.

\subsection{Scalable Media Stream}

There has been a lot of extensive research on scalable video transport and resource management over mobile wireless networks, focusing on unicast services [6] 7] [ The fine granularity scalable (FGS) video coding scheme 9] in MPEG-4 [10] encodes video frames into multiple layers, including a base layer of video of relatively lower quality and several enhancement layers containing increasingly more detailed data that are used to enhance the base layer and hence to improve the quality of the final video. The progressive fine granularity scalable (PFGS) coding scheme 11] represents an advance on the FGS scheme.

These scalable video coding schemes feature a layered bit-stream structure in which different layers have different levels of importance. The base layer stream is very sensitive to channel errors. If the decoder finds any errors in the lower layers, the higher layers of the current frame are discarded whether they are correct or not. If they go undetected, errors in the base layer will propagate to the start of the next group of pictures (GOP) and cause serious drifting problems in the higher layers of the following frames. While accuracy in the base layer is essential for decoding video streams, the enhancement layers are more tolerant of channel errors. Errors in the enhancement layers corrupt video quality only in the current frame and a few subsequent frames, and the deterioration is not as noticeable as that caused by errors in the base layer. Because of this layered bitstream structure, it seems reasonable that the more important layers should be provided with more protection against errors than the less important layers. But the error recovery scheme in the current BCMCS standard makes no allowance 
for the different value of the different layers, and all are equally likely to be damaged during transmission. Our adaptive framework confronts this problem, and has the following key features:

- Graceful quality degradation: unlike non-scalable video, scalable video can adapt its representation to bandwidth variations. When the network has to drop packets, it does so with awareness of the video that the data represents. As a result, perceptual quality is gracefully degraded even under severe channel conditions.

- Efficiency: when there is excess bandwidth, it is used efficiently in a way that maximizes the perceptual quality or the revenue.

- Fairness: the resource can be shared to enhance the average playback quality. We propose three policies to share slot resources efficiently.

- Guaranteed delivery of the base layer: the base layer stream is guaranteed by giving it more opportunities for retransmission. This avoids abrupt drops in service quality.

\section{The Proposed Scheme}

From an established service scenario [5], we can infer that current static scheduling algorithms cannot adapt to an environment in which broadcast flows start and stop in part of the system, affecting the resources available. For example, when a video stream is added, empty slots must be found to schedule this new content. Because the transmission rates of video streams differ from each other, the slot periods of these video streams will also differ. Thus, the slots used by different streams may overlap, which can cause problems in servicing them. Additionally, we can infer that, if all slots are allocated for predetermined video streams, additional video streams or newly created asynchronous video streams (newsflashes or other irregular content) that are not predetermined would never be serviced. This inefficient slot utilization is exacerbated by the parity data required for Reed-Solomon coding. In addition, the current scheduling scheme makes it impossible to change the quality of a video stream in accordance with bandwidth variations. This motivates us to deploy a dynamic scheduling algorithm. Specifically, we propose to apply the EDF real-time scheduling algorithm, which endeavors to deliver packets before their deadlines, together with retransmission to maximize the slot utilization in BCMCS.

When many video servers provide multimedia streams, each BCMCS flow is continually split into packets which then wait to be serviced. If a mobile node cannot receive a packet, that packet is reported as lost or damaged to the base station, via the reverse unicast channel. Because successful receipt of a broadcast packet will give satisfaction to more users than receipt of a retransmitted packet, the scheduler gives a higher priority to broadcast packets than to retransmitted packets, and base-layer packets are given higher priority than enhancement-layer packets. Thus, broadcast packets will be transmitted earlier, giving them more chance of retransmission in case of loss. Then, if any slots are still available, base-layer packets are transmitted, and finally enhancement-layer packets. 
Table 1. Parameters used in this paper

\begin{tabular}{cl}
\hline Parameter & Description \\
\hline$b_{i}$ & Bit rate of each BCMCS IP flow (kbps) \\
$p_{i}$ & Period of $\tau_{i}$ in units of slots \\
$R_{i}$ & Buffer requirement of each BCMCS IP flow \\
$D_{i}$ & Relative deadline slot for the periodic multicast video frame $\tau_{i}$ \\
$D_{a k}$ & Deadline slot for retransmitted packets $\bar{\tau}_{k}$ \\
$F_{i}$ & Size of transmitted data during one period for each video stream \\
$e_{i}$ & Number of slots required to forward video stream during one period \\
$\bar{e}_{r}$ & Number of slots required to retransmit lost packets of $\bar{\tau}_{r}$ \\
$M_{i}$ & Number of bytes that can be forwarded in one slot (data payload) \\
$U_{P}$ & Utilization of periodic video frames \\
$U_{c u r}$ & Current slot utilization \\
$\tau_{i}$ & Periodic broadcast video frame \\
$\bar{\tau}_{k}$ & Aperiodic retransmitted video frame (packets) \\
$A_{k}$ & Arrival time of $\bar{\tau}_{k}$ \\
$N$ & Total number of mobile nodes in a cell \\
$N_{\tau_{i}}$ & Number of mobile nodes registered for video flow $\tau_{i}$ \\
\hline
\end{tabular}

Admission control and resource allocation are tightly related to the packet scheduling algorithm. In our scheme, the admission of newly created video frames and retransmitted packets is allowed if sufficient resources for the adequate delivery of data to a mobile node are available. In the EDF scheduling algorithm, resource availability is checked by a utilization bound test. In our scheme, admission is also controlled by a utilization bound test, as follows: Suppose there are $n$ BCMCS flows currently being serviced in the cell and the bit-rate of each BCMCS flow is $b_{i} \mathrm{kbps}$. Then the scheduler must periodically transmit $b_{i}$ kbits per second, since BCMCS flow corresponds to a periodic task in a realtime system. Thus, each flow has the period $p_{i}$. If the buffer requirement $\left(R_{i}\right)$ to guarantee continuous playback is $K * p_{i}$, then the relative deadline slot is $\left(K * p_{i}\right) / \varphi$ where $\varphi$ is the fundamental timing unit of a slot and has the value of $1.67 \mathrm{~ms}$. Using the transmission scheme in cdma2000 $1 \mathrm{xEV}-\mathrm{DO}, e_{i}$ is determined by $\left\lfloor b_{i} / M_{i}\right\rfloor$. Thus the admission of a new multicast video stream $\left(\tau_{j}\right)$ is decided using the following equation [3]:

$$
U_{P}=\sum_{i=0}^{n-1} \frac{e_{i}}{\operatorname{Min}\left(p_{i}, D_{i}\right)}+\frac{e_{j}}{p_{j}} \leq 1 .
$$

If $U_{P}>1$, the scheduler adjust the scalability of existing video streams to permit the new video frame. We consider three policies to adjust the scalability of each video stream, experimentally comparing their performance with respect to the peak signal-to-noise ratio (PSNR) value:

- Policy 1 (Fair degradation): the appearance of a new video stream produces equal quality degradation in all existing flows. 
- Policy 2 (Victim degradation): victim flows are selected for adjustment to permit the newly requested video flow.

- Policy 3 (Proportionally fair degradation): the degradation in quality of each existing flow is inversely proportional to the number of subscribers that it has.

There may also be packets for retransmission, generating aperiodic tasks which increase slot utilization in proportion to their number. If the current slot utilization is $U_{c u r}$, then the average slot utilization by retransmitted packets for one mobile node will be $\left(U_{\text {cur }}-U_{P}\right) / N$. When a request to service new video flow is made, the bit-rates of existing flows have to be adjusted. A reserve capacity $\left(U_{\text {reserved }}\right)$ of $\left(U_{\text {cur }}-U_{P}\right) *\left(1+N_{\tau_{j}} / N\right)$ must be provided for retransmitted packets, which leaves $1-U_{\text {reserved }}$ to be allocated to periodic broadcast flows, by application of the policies just discussed.

Using the first policy, when $m$ new video flows are requested, and the number of available slots is insufficient, the bit-rate of each flow $i$ is degraded in an egalitarian manner. The degraded bit-rate is given as follows:

$$
\dot{b}_{i}^{\prime}=\left\lfloor b_{i} * \frac{1-U_{\text {reserved }}}{U_{\text {cur }}+\sum_{j=0}^{m} \frac{e_{j}}{p_{j}}}\right\rfloor .
$$

Using the second policy, victims are selected for a degraded flow. If the bit-rate of the first flow drops below the base-layer bit-rate, then another victim is selected from among those flows which are not already victims: we choose the one with the smallest number of subscribers. The bit-rates of additional victim flows are degraded in a similar manner to the first. Using the third policy, the bit-rate is degraded in proportion to the number of subscribers. If the bit-rate of the flow with the lowest subscriber count falls below the base-layer bit-rate, then the bit-rate of that flow is fixed at the minimum value, and the other flows are scaled down appropriately. The adjusted bit rate $b_{i}^{\prime}$ of video flow $i$ is given as follows:

$$
\begin{gathered}
\text { Degradation }=\left(\sum_{i=0}^{n-1} b_{i}+\sum_{n=0}^{j=m-1} b_{j}\right)\left(1-\frac{1-U_{\text {reserved }}}{U_{\text {cur }}+\sum_{j=0}^{m} \frac{e_{j}}{p_{j}}}\right) \\
b_{i}^{\prime}=\text { Degradation } * w_{i},
\end{gathered}
$$

where $w_{i}$ is a weight which is inversely proportional to the mobile node count of video flow $i$, selected from among the values $\frac{N_{\tau_{i}}}{N}, \frac{N_{\tau_{j}}}{N}(0 \leq i \leq n-1$, $0 \leq j \leq m-1)$.

Admission of newly generated retransmission packets can be decided in the following way [12]13]: Suppose the aperiodic task set $Q(t)=$ $\left\{\bar{\tau}_{0}, \bar{\tau}_{1}, \bar{\tau}_{2}, \ldots \bar{\tau}_{k}, \ldots\right\}$ and the periodic task set $P=\left\{\tau_{0}, \tau_{1}, \tau_{2}, \ldots \tau_{i-1}\right\}$ are to be scheduled by the EDF algorithm. For $\bar{\tau}_{k} \in Q(t), \bar{e}_{k}(t)$ is defined as the remaining execution time of $\bar{\tau}_{k}$ at time $t$, and we define the set $S$ to be the union of $Q(t)$ and $P$. For all $\bar{\tau}_{k} \in Q(t), S(t, \infty)$ is schedulable if and only if $\forall k, U_{S(t, \infty)}\left(\bar{\tau}_{k}\right) \leq 1$, 
where

$$
U_{S(t, \infty)}\left(\bar{\tau}_{k}\right)=\frac{\sum_{i=0}^{n-1} e_{i}(t)+\sum_{\bar{\tau}_{r} \in Q\left((t, \infty), H P\left(D_{a k}\right)\right)} \bar{e}_{r}(t)}{D_{a k}-t} .
$$

The term $H P\left(D_{a k}\right)$ represents 'higher priority'. If $\bar{\tau}_{k}$ is included in the baselayer packets, then $\bar{\tau}_{j}$ are tasks which is included in the base-layer packets and have an earlier deadline than $D_{a k}$. If $\bar{\tau}_{k}$ is included in the enhancement-layer packets, then all retransmission packets corresponding to the base layer or the enhancement layer, with deadlines earlier than $D_{a k}$, are included in $\operatorname{HP}\left(D_{a k}\right)$. Thus, base-layer packets have a higher priority than enhancement-layer packets. If two packets correspond to the same layer, the packet with the earlier deadline has the higher priority. Using Equation (5), we might now expect to be able to determine the feasibility of a new retransmission request. However this test is actually impossible because an infinite number of tasks $\left(Q\left((t, \infty), H P\left(D_{a k}\right)\right)\right)$ would have to be considered. So we use the following recursive method to calculate an upper bound on the utilization.

A task set $S(t, \infty), Q(t)=\left\{\bar{\tau}_{1}, \bar{\tau}_{2}, \bar{\tau}_{3}, \ldots, \bar{\tau}_{k-1}, \bar{\tau}_{k}, \ldots\right\}$ is sorted by priority. Thus, $\bar{\tau}_{k-1}$ has a higher priority than $\bar{\tau}_{k}$, and an aperiodic task $\bar{\tau}_{k} \in Q(t)$ is schedulable when $\bar{U}\left(\overline{\tau_{k}}\right) \leq 1$. The virtual finish time $f_{k}$ is defined as the sum of the execution times (number of slots required) of all periodic tasks and of the aperiodic tasks that have a higher priority than $\bar{\tau}_{k}$ during the time period $\left[A_{k}, D_{a k}\right]$. Thus

$$
\begin{gathered}
U\left(\bar{\tau}_{k}\right) \leq \frac{\max \left\{f_{k-1}-A_{k}, 0\right\}+\bar{e}_{k}+U_{P} *\left(D_{a k}-D_{a(k-1)}\right)}{D_{a k}-A_{k}}=\bar{U}\left(\bar{\tau}_{k}\right) \\
f_{k-1}=A_{k-1}+U\left(\bar{\tau}_{k-1}\right) * d_{k-1} .
\end{gathered}
$$

Using these equations while fixing $U_{k}$ and $F_{k}$, the schedule can be analyzed within constant time. Hence, we can ignore the admission control overhead.

\section{Performance Evaluation}

\subsection{Simulation Model}

Fig. 1 shows our experimental architecture for broadcasting scalable video from a base station to mobile nodes. Admission control and resource reservation for retransmitted packets are two of the major factors in controlling the transmission rate, as explained in the previous section. If a request is accepted, rate-controlled video packets are scheduled in an EDF manner.

We conducted experiments using the Foreman testbench video sequences streamed at 30 frames per second with a total of ten thousand frames. Each video stream is handled with our reference MPEG-4 FGS codec, which is derived from the framework of the European ACTS Project Mobile Multimedia Systems (MoMuSys) 15] and modified for our purpose. It consists of a $120 \mathrm{kbps}$ base-layer bit-rate and a $100 \mathrm{kbps}$ enhancement-layer bit-rate with 120 levels 


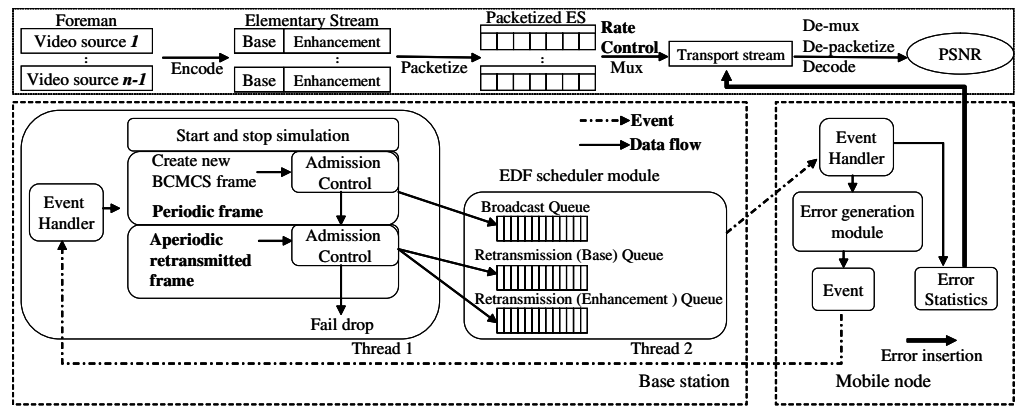

Fig. 1. Simulation architecture

(1 kbps unit). All video streams are sent through channel coding and packetized before being transferred via a cdma2000 1xEV-DO physical slot. In the experiment, we used QPSK modulation with a $1228.8 \mathrm{kbps}$ data-rate forward channel.

At the mobile node, successful receipt of the video packet is determined by an error generation module, using the simple threshold model suggested by Zorzi 16. When an error occurs, this module may subsequently request retransmission of packets by means of a retransmission event generated by the event generation module. The affected packets are then inserted into a queue by an event handler in the base station, where they wait to be serviced by the scheduler. In our simulation environment, the air channel is simulated by a two-state Markov model, which can simulate the error sequences generated by data transmission channels. These errors occur in clusters or bursts with relatively long error-free intervals between them. In the following equations, $1-\alpha$ (or $\beta$ ) is the probability that the $i$-th packet block is successfully transmitted, given that the $(i-1)$-th block was successful (or unsuccessful). Fading in the air channel is assumed to have a Rayleigh distribution. The steady-state error rate $\varepsilon$ is then obtained as follows:

$$
\varepsilon=\frac{\alpha}{\alpha+\beta}
$$

If the Rayleigh fading margin is $F$, the steady-state error rate can be given as

$$
\varepsilon=1-e^{-\frac{1}{F}}
$$

We can now derive the values of $\alpha$ and $\beta$ from the following equations, and from Equations (8) and (9). $F$ is the fading margin and the parameter $f_{d} N_{B L} T$ (the Doppler frequency normalized to the data rate where $N_{B L}$ represent the block (packet) length) is taken as 0.02. Burst is the average length of packet errors and is given by $1 / \beta$, where

$$
\beta=\frac{Q(\theta, \rho \theta)-Q(\rho \theta, \theta)}{e^{\frac{1}{F}}-1} \text {, and } \theta=\sqrt{\frac{2 / F}{1-\rho^{2}}}
$$


The term $\rho$ is the correlation coefficient of two samples of the complex Gaussian fading process, and is expressed as $\rho=J_{0}\left(2 \pi f_{d} T\right)$. Additionally,

$$
Q(x, y)=\int_{y}^{\infty} e^{-\frac{x^{2}+\kappa^{2}}{2}} I_{0}(x \kappa) w d \kappa
$$

is the Marcum- $Q$ function. Thus the relationship between the steady-state error rate and the Markov parameter can be represented as

$$
\begin{gathered}
\beta=\frac{1-\varepsilon}{\varepsilon}[Q(\theta, \rho \theta)-Q(\rho \theta, \theta)], \text { where } \\
\theta=\sqrt{\frac{-2 \log (1-\varepsilon)}{1-J_{0}^{2}\left(2 \pi f_{d} N_{B L} T\right)}} .
\end{gathered}
$$

We now compare our retransmission model with the current Reed-Solomon-based BCMCS error correction scheme. The code $(16,12,4)$ is used in our experiments. To evaluate two error recovery schemes, errors are injected into the original transport stream and the PSNR of the resulting video stream is calculated to estimate the quality of a reconstructed image compared with an original image. Finally, we compare the current Reed-Solomon-based static scheduling scheme with the proposed retransmission-based dynamic scheduling scheme with respect to PSNR.

\subsection{Experimental Result}

In our experiment, we used two sets of data: Set 1 is $\left\{\left(\tau_{i}, N_{\tau_{i}}\right) \mid\left(\tau_{0}, 5\right),\left(\tau_{1}, 2\right),\left(\tau_{3}\right.\right.$, $\left.5),\left(\tau_{4}, 12\right),\left(\tau_{5}, 20\right)\right\},\left(\tau_{1}\right.$ is a new video flow $)$, Set 2 is $\left\{\left(\tau_{i}, N_{\tau_{i}}\right) \mid\left(\tau_{0}, 5\right),\left(\tau_{1}, 2\right),\left(\tau_{2}\right.\right.$, $\left.2),\left(\tau_{3}, 5\right),\left(\tau_{4}, 12\right),\left(\tau_{5}, 20\right)\right\}$ and $\tau_{1}$ and $\tau_{2}$ are new video flows. $U_{B C M C S}$ is the slot utilization when frames are encoded by Reed-Solomon coding, and $U_{\text {Proposed }}$ is the slot utilization when the proposed retransmission scheme is used. Before any new video flows are created, $U_{B C M C S}=1$, which means that no available slot is left. But $U_{\text {Proposed }}=0.75$, because $25 \%$ of the slots are saved in comparison to the current scheme, which corresponds to the space that would have been used by parity data. These saved slots are used to recover lost packets by retransmission.

Fig. 2 shows the average PSNR of all mobile nodes using data Sets 1 and 2 when the physical-layer packet error-rates $\left(P E R_{\text {physical }}\right)$ are $1 \%$ and $5 \%$. We sampled from Frame 2000 to Frame 3000 of Set 1, and from Frame 3000 to 4000 of Set 2. From these graphs, we can see that average PSNR improves when a new video flow is admitted to a cell and bandwidth is shared with the current existing video flows. We contrast this with the situation in the current scheme, where the new flow would never be admitted. The gap between the two curves (current scheme vs. proposed scheme) in the graph increases as more flows share the bandwidth. The large fluctuations in the lower curve come from the failure of base-layer packets, an effect which is exacerbated as the physical-layer packet error-rate $\left(P E R_{\text {physical }}\right)$ increases. When a base-layer packet is broken, the video quality is abruptly degraded. 


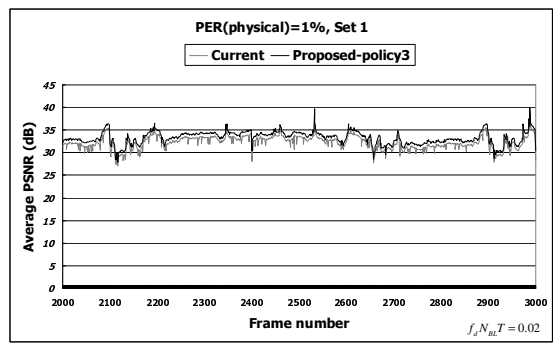

(a) PER $1 \%$, Set 1

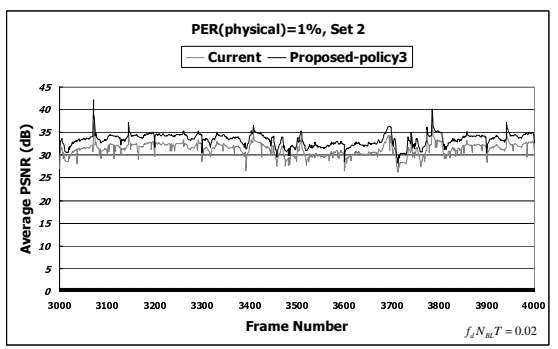

(c) PER 1\%, Set 2

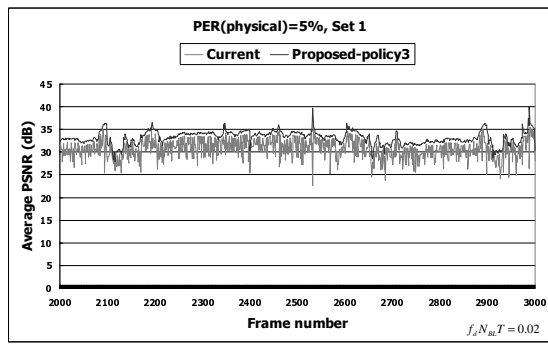

(b) PER 5\%, Set 1

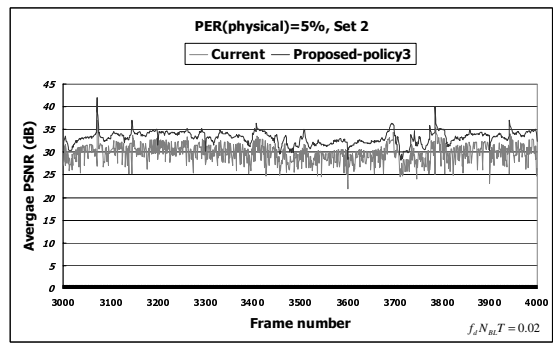

(d) PER 5\%, Set 2

Fig. 2. Average PSNR of all mobile nodes for Set 1 and Set 2

In Fig. 3(a) and 3(b), three proposed policies are compared with each other and with the current scheme using the experimental data Sets 1 and 2. As a new video flow is admitted, the average PSNR per flow is somewhat degraded. But we can see that the overall average is much improved compared to the current scheme. When the $P E R_{\text {physical }}$ is $5 \%$ and Policy 3 is used, the overall average improves as much as $18.7 \%$ compared to the current scheme, as shown in Fig. 3. b). We can improve the average playback quality by considering the distribution of mobile nodes which subscribe to each video flows although it is a relatively small effect compared to quality adjustment and packet retransmission. Because the dispersion of subscriber numbers in our experimental set is large, the third policy produces the best quality. The average PSNR using Policy 3 for Set 2 is approximately 0.05 $\mathrm{dB}$ higher than that using Policy 1. Using Set 1, however, when the $P E R_{\text {physical }}$ is $3 \%$ or $5 \%$, Policy 2 gives a slightly better result. The final result will also depend to an extent on the dispersion of subscriber numbers and on the shape of the curve relating bit-rate to PSNR, an observation which can be used to optimize the resource allocation to maximize the average playback quality.

Fig. 4 compares the average PSNR of nodes for each video flow when the $P E R_{\text {physical }}$ is $3 \%$. As a new flows are admitted, the bit-rate of each video flow is adjusted according to the three proposed policies. In the current scheme, new flows (Flow 1,2) can never be serviced because existing flows already occupy 


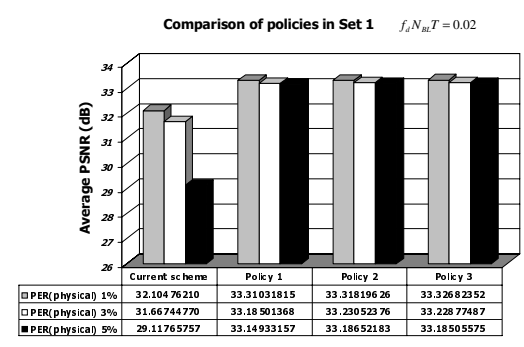

(a) Set 1

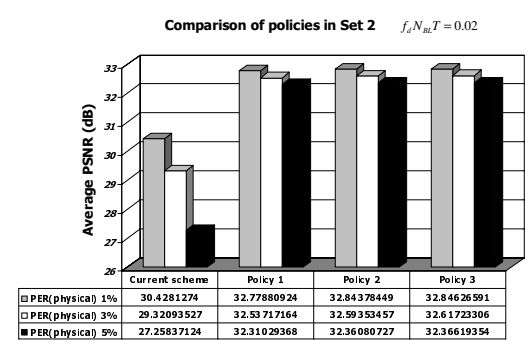

(b) Set 2

Fig. 3. Comparison of policies

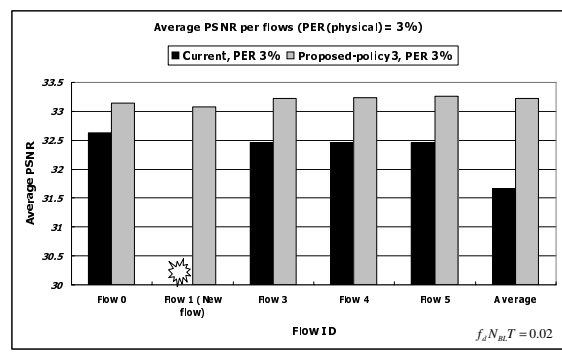

(a) Set 1

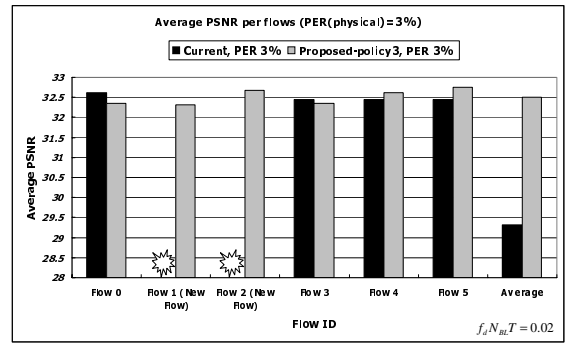

(b) Set 2

Fig. 4. Average of PSNR with respect to flows

all the slots, which are allocated statically. Thus the average PSNR of each new video flow in Fig. 4 is zero, which degrades the average PSNR. This degradation grows as the number of subscribers to new video flows increases.

\section{Conclusion}

We have proposed a dynamic scheduling algorithm based on EDF to handle the situation where broadcast channels change dynamically. Our algorithm can adapt to a dynamic context where the bandwidth requirement of all video flows fluctuate, and it also meets the real-time requirement of broadcast streams. In addition, we prevent abrupt quality degradation of broadcast streams by deploying a retransmission scheme, instead of the Reed-Solomon coding used in the current BCMCS, to cope with poor channel condition. By dynamically adjusting the scalability of the video streams that are already being serviced, even when no available slots remain, new video streams can be admitted, although the playback quality is somewhat degraded. Extensive simulation has shown the efficiency of our scheme compared with that used by the current BCMCS. By protecting the base layer by means of retransmission, we can avoid abrupt drops 
in service quality. Our experimental results show that the average PSNR of all mobile nodes in our proposed scheme is higher than in the current BCMCS scheme. In addition, by considering the distribution of subscribers across the video flows, we can further improve the average playback quality.

\section{References}

1. P. Agashe, R. Rezaiifar, P. Bender and QUALCOMM, "Cdma2000 high rate broadcast packet data air interface design," IEEE Communications Magazine, vol. 42, no. 2, pp. 83-89, February 2004.

2. 3GPP2 C.S0024 v3.0, "Cdma2000 high rate packet data air interface specification," December 2001.

3. F. Cottet, J. Delacroix, C. Kaiser and Z. Mammeri, Scheduling in Real-Time Systems, Wiley, pp. 31-32, October 2002.

4. G. D. Mandyam and Y. C. Tseng, "Packet scheduling in CDMA systems based on power control feedback," Proc. IEEE International Conference on Communications, vol. 9, pp. 2877-2881.

5. J. Wang, R. Sinnarajaj, T. Chen, Y. Wei, E. Tiedemann and QUALCOMM, "Broadcast and multicast services in cdma2000," IEEE Communications Magazine, vol.42, no. 2, pp. 76-82, February 2004.

6. D. Wu, T. Hou and Y.-Q. Zhang, "Scalable video transport over wireless IP networks," Proc. 11th IEEE International Symposium on Personal, Indoor and Mobile Radio Communication, vol. 2, pp. 1185-1191, September 2000.

7. C.-H. Yeh, "Scalable, adaptive, and reliable resource management in highspeed and mobile networks," Proc. 10th Conference on Computer Communications and Networks, pp. 182-189, October 2001.

8. K. Gao, W. Gao, S. He, P. Gao and Y. Zhang, "Real-time scheduling on scalable media stream delivery", Proc. International Symposium on Circuits and Systems, vol. 2, pp. 25-28, May 2003.

9. W. Li, "Overview of fine granularity scalability in MPEG-4 video standard," IEEE Transactions on Circuits and Systems for Video Technology, vol. 11, no. 3, pp. 301317, March 2001.

10. ISO/IEC 14496-2, "Coding of audio-visual objects - part2: visual," May, 2004.

11. F. Wu, S. Li and Y. Q. Zhang, "A framework for efficient progressive fine granularity scalable video coding," IEEE Transactions on Circuits and Systems for Video Technology, vol. 11, no. 3, pp. 332-344, March 2001.

12. J. Park, M. Ryu and S. Hong, "Utilization demand analysis: An integrated admission control aproach for a mixed set of periodic and aperiodic tasks," Proc. Korea Information Science Society Spring Conferences, pp. 391-393, April 2004.

13. M. Spuri and G. Buttazzo, "Scheduling aperiodic tasks in dynamic priority systems," Real-Time Systems, vol. 10, no. 2, pp. 179-210, March 1996.

14. B. K. Choi, D. Xuan, R. Bettati, W. Zhao and C. Li, "Utilization-based admission control for scalable real-time communication," Real-Time Systems, vol. 24, no. 2, pp. 171-202, March 2003.

15. A. Pearmain, A. Carvalho, A. Hamosfakidis and J. Cosmas, "The MoMuSys MPEG-4 mobile multimedia terminal," Proc. 3rd ACTS Mobile Summit Conference, pp. 224-229, June 1998.

16. M. Zorzi, R. R. Rao and L. B. Milstein, "Error statistics in data transmission over fading channels," IEEE Transactions on Communication Communications, vol. 46, no. 11, pp. 1468-1477, November 1998. 